\title{
Physical Activities and Second Language Proficiencies; A Systematic Review
}

\author{
Fatona Suraya ${ }^{1}$, Hendi Pratama ${ }^{2}$, Puput Arfiandhani ${ }^{3}$ \\ \{suraya@mail.unnes.ac.id ${ }^{1}$, hendi.pratama@mail.unnes.ac.id ${ }^{2}$, puput.arfiandhani@fpb.umy.ac.id ${ }^{3}$ \} \\ Universitas Negeri Semarang, Semarang, Indonesia ${ }^{1,2}$, \\ Universitas Muhammadiyah Yogyakarta, Bantul, Indonesia ${ }^{3}$
}

\begin{abstract}
Research has shown that physical activities provide a positive effect on cognitive performance, including short-term and long-term memory. however few have focused on its effect on the second language (L2). This paper is a systematic literature review (SLR) paper that aims to identify, assess, and interpret related research papers to gain a better understanding of the positive possible effect of several types of physical activities toward L2 performances. Eight different papers published between the years 2007 to 2019 are analyzed in terms of the physical activities, the research method, and the L2 domain being affected. It suggest that there is a positive association between physical activities and L2 learning. The positive effect are being discussed.
\end{abstract}

Keywords: physical activities, second language performance, systematic literature review

\section{Introduction}

Research has shown that physical activities provide a positive effect on cognitive performance[1-4], including short-term and long-term memory[5] however few have focused on its effect on the second language (L2). This systematic literature review tries to seek the trend of research focusing on the effect of physical activities to L2 performances and analyze on what area of L2 performance benefit more from the physical activities by examining several published paper in the field. Physical activity (PA) defines as any bodily movement produced by skeletal muscles that require energy expenditure, including playing. Second language (L2) defines as any language that a person uses other than a first or native language (L1). This study aims to identify sample characteristics, research methods, and type of physical activities intervention being implemented in research based on physical activities and L2 performance as well as exploring the language domain being affected by the physical activities.

\section{Methods}

\subsection{Review method}


This study employs a systematic literature review as its methodical approach. According to Moher, a systematic review is a process of reviewing which focus on answering a formulated question that employs systematic and explicit methods to specifically pick and choose relevant researches and to gather and analyze data from papers which are selected to be reviewed [6] This study follows the common review protocol of a systematic review as explained in the article of Five steps to conducting a systematic review namely framing the research questions, identifying relevant publications, asssessing the quality of studies, summarizing the evidence, and Interpreting the findings [7].

\subsection{Framing research questions}

The research question in this study aimed to maintain the focus of the review. The question guiding this study is what kinds of research methods and physical activities intervention being used in the studies on physical activities and L2 performance? How do the physical activities affect the L2 performance?

\subsection{Identifying relevant publication}

To gather as many relevant citations as possible, a variety of second/foreign language, sports, physical activities, language assessment were searched to identify primary studies of the relation of physical activities toward L2 proficiencies. A wide range of internet searching engines was supplemented by hand searching to provide references. Here is the list of the digital database searched: google scholar, Ebsco, ScienceDirect, Asian-EFL-journal, Researchgate, Natecla, journals-PLoS, PubMed. The last search was conducted on July 9, 2020. The inclusion and exclusion criteria modifying a systemic review research model on engineering research[8] are explained in table 1.

Table 1. Inclusion and exclusion criteria

\begin{tabular}{ll}
\hline Inclusion & research in an academic setting using large and small scale data sets \\
& research discussing and comparing the various model of motor/physical \\
activities intervention toward L2 performance & For research which has both conference and journal version, only the \\
journal version is included & Research within the last 15 years (2006-2020) \\
\hline Exclusion & research in a non academic setting \\
& Research not written in English \\
& The article that focused on physical activities and neurocognitive function \\
& The article that is not published in a journal \\
\hline
\end{tabular}

Sofware package EndNote was employed to store and manage the search result. This effort resulted in 51 related articles. Their potential relevance was examined according to the inclusion and exclusion criteria of table 2. Of 31 articles were excluded as irrelevant. The full papers of the remaining 20 articles were assessed to select those primary studies in physical activity intervention and L2 performance. This selection excluded 13 studies and left 8 articles in the review. The article comes from 5 countries, published in 4 different journals between the year 2007 to 2019 . 


\subsection{Assessing study quality}

In addition to the inclusion and exclusion criteria in table 2, the quality of primary studies, and their relevance to the research questions were considered. The complete list of selected studies is presented in table 3. Selected Article whereas the quality assessment of studies on physical activities and L2 performance are explained in table 3.

Table 2. Quality assessment of the study

\begin{tabular}{|c|c|c|}
\hline Quality categories & High & Moderate \\
\hline Population & More than 50 & Between 50 to 12 \\
\hline Intervention & $\begin{array}{l}\text { Physical activities and language } \\
\text { input with equal proportion }\end{array}$ & $\begin{array}{l}\text { Physical activities and language } \\
\text { input with an inadequate } \\
\text { proportion }\end{array}$ \\
\hline Comparison & various type of physical activities & single types of Physical activities \\
\hline & $\begin{array}{l}\text { and various aspect toward L2 } \\
\text { performances }\end{array}$ & $\begin{array}{l}\text { toward a single aspect of L2 } \\
\text { performances }\end{array}$ \\
\hline Outcomes & $\begin{array}{l}\text { Adjustments for at least three } \\
\text { indicators }\end{array}$ & $\begin{array}{l}\text { Adjustment for at least one } \\
\text { indicator }\end{array}$ \\
\hline Context & $\begin{array}{l}\text { Study in early childhood } \\
\text { education and adults }\end{array}$ & Study in children in general \\
\hline
\end{tabular}

\subsection{Summarizing the Evidence}

This paper restricts to summarizing the finding narratively. The association between physical activities and L2 performances, in general, was examined in 8 studies. The details of the summary are discussed in chapter 3 Result. Whereas the last stages of review namely interpreting the finding will be clearly explained in chapter 4 discussion.

\section{Results and discussions}

Paper by Winter [9] on High impact running improves learning examines the learning performance examine 27 male athletes directly after two conditions: high impact anaerobic sprints and low impact aerobic running. It involves an immediate and long term study. The participants were given a neuropsychological test which was analyzed using the Pearson correlation coefficient and fitness test to measure their physical fitness level. The activity followed by exercise intervention which divided into three conditions namely relax (control group), moderate and intense as the experiment group. The individual heart rate was taken into consideration before and after each intervention. Immediately after the intervention, the experiment group rated their perceived exertion on the Borg-Scale. The learning intervention given in form of correct pairing, a visually presented daily object, and a novel word (e.g., car and /glump/) and the participant need to decide correct or not by pressing one of two keys with their right hand. Of 600 training trials were prepared for the participants. Retention was given a week after and 6-After 8 months post, respectively. The treatment showed a positive effect on learning. The intense subject condition learns $20 \%$ faster after two sprints of less than 3 min each compared to moderate and relaxes conditions. It contends that quick exerciseinitiated consequences for a mind complex learning task straight with neurophysiological correlates analysis(changes in peripheral catecholamine or BDNF levels) in people. The 
analysts contend that increasingly supported BDNF levels during learning (levels after extraordinary exercise less levels subsequent to learning) were identified with better transient learning achievement, and outright dopamine and epinephrine levels after serious exercise was identified with better middle of the road (dopamine) and long haul (epinephrine) maintenances of the novel vocabulary. Thus, exercise-induced learning improvement for short-term memory and long term memory in terms of word memory process and novel word recognition. However, this paper was only focused on male athletes which as we could assume have high motivation for passing the physical intervention.

The next article on the Physical activity and mental performance in preadolescents: Effects of acute exercise on free-recall memory were also testing word memory process in a visual test form[5]. The physical exercise involved were team games and circuit training started by warming up activities and closed by cooling down activities that were conducted during the Physical Education (PE) lesson. The third group was the baseline group which did not proceed by any lesson. There were two conditions of free-recall test namely immediate and delay recall. The intensity of the exercise during the PE lesson was observed by recording Heart Rates (HR) and rating of perceived exertion (RPE). RPE was recorded after the PE lesson and before starting the memory test. The data were analyzed using statistical analysis. The result of the study explained that for immediate recall group following team game score higher for both primacy and recency portions than in the baseline session. On the other hand, delayed recall groups score higher in the recency portion after aerobic training activities and team games. The research suggests that acute submaximal exercise facilitate memory storage in term of free-recall memory of random words. Further, the study discussed an exercise-induced benefit for memory performance that cannot be confounded with learning effects, it does improve the mastery of vocabulary but does not equally improve the learning. Interestingly, this study refers to the previous study discussed in this paper [9] arguing that the short-term effect of exercise may also have mental health outcomes in the long-term memory through examining BDNF levels during learning after exercise.

Article by Schmidt-Kassow's et.al discussing exercising during learning improves vocabulary acquisition[10] The study aimed to clarify whether the dynamic movement during learning stimulates by physical activities influences verbal learning compared to learning in static condition. The study involved two groups, spinning group, and passive groups, and it measured behavioral and electrophysiological responses to memory materials. They were given matching and miss matching words and were asked to analyze the correct one. The study indicates that simultaneous physical activity during foreign language vocabulary learning facilitates the memorization of new items. Interestingly, this study strengthens the previous study on comparing different learning conditions (static condition versus dynamic condition) toward the vocabulary memorization [9]. In addition, this study examines the longterm effect of regular physical activity during learning toward the learning process and was able to prove the hypothesis that the spinning group showed significantly better performance of foreign language vocabulary testing each day even after three weeks of learning. Subjects who were physically active in the encoding phase have a larger N400 effect and have better performance in vocabulary tests. However, this study involved a very small sample of 12 healthy subjects, 4 males and 8 females.

The next article examining the use of Total Physical Response (TPR ) method in Early childhood foreign language[11]. TPR is merging speech and action coordination in teaching (the foreign language) through physical activity. It involves no texts in the teaching environment but there is teaching via games, dance-songs, stories with actions to attracts 
children's attention. The paper discussed the positive view on implementing TPR to teach early childhood foreign language classrooms using a narrative qualitative approach. It argues that TPR activities that are full of action benefit those kinesthetic learners. The objective of TPR is not in writing or reading but more likely to speaking and listening as the aim is to introduce new vocabulary to children in a more meaningful context and invite children to respond (verbally or non verbally).

Research on integrated gesture and physical activities on early childhood's foreign language vocabulary learning differentiate the movement type into part-body movement (gesture) and whole-body movement (physical activity) in given foreign language vocabulary tasks [12]. It assumed that emerging movement into a cognitive learning task may be beneficial for learning because of its cognitive and psychological aspect, meaning that enacting the words through physical exercises will give positive cognitive effects. Four conditions were implemented namely integrated physical exercise, non-integrated physical exercise, and gesturing condition and conventional condition. In the integrated physical exercise, children learning and acting new words that are related to the physical activity in the class. In the non-integrated group, the children get the same exercise intensity but not related to the learning task. In the gesturing condition, the students remained seated while acting the targeted words. Whereas in conventional conditions, the children repeated the target words continuously without any gesture or moving. Of 111 children were introduced to 14 foreign words in 4-week teaching. They were tested for their memory of words during the program, immediately after the program and weeks after the program (follow up) using free-recall and cued recall tests. Mixed 4 conditions were analyzed $a=$ based on ANOVA with free-recall and cued-recall as the dependent variables. Whereas condition between subject and time of testing within-subject as the independent variables. The result confirmed the hypothesis that integrated physical exercise where the children learn the unfamiliar foreign vocabulary while performing related physical exercise (to emphasize the meaning of the word), produce the highest immediate and delayed retention performance, in other words, it produces the highest learning outcome. It revealed that the integrated condition outperformed other all other 3 conditions either during, immediately after, and six-week after the learning.

While other studies discussed the effect of physical activities toward L2 performance for beginner learner who has never immerse in the L2 learning previously, this study by Liu and colleagues[13] try to challenge the previous study by testing possible constructive effects of physical activity toward L2 learner who has already reached some level of language competence. Additionally, this research also ahead of other research in the field in terms of linguistic competence being observed which is not only discussing the word level (employing Word-Picture Verification task) but sentence level (employing semantic judgment task). 40 samples were involved and divided into two control groups and two experiment groups with equal distribution of males and females. Both of the groups have a similar level of L2 proficiency. The experiment group was assigned to spin a bicycle during the learning phase when they were given a word-picture presentation. The exercise intensity was monitored and controlled. Physical activities benefit the beginner level of L2 students in learning a set of unfamiliar vocabulary. Moreover, their performance continuously immersed in the semantic level as indicated by the result of a Semantic Judgment task. Interestingly, a temporal asymmetry between the lexical and the semantic level emerges, with the difference between the experimental and control group emerging from the 1st testing session at the lexical level but after several weeks the participant takes re-test/ delayed testing the result were higher for experiment group at the sentence processing level. 
The next study by kruger [14] is a pilot study to sixty-one young refugees joining physical education (PE) class with a modification of physical activity within the context of primary PE games with L2 learning activities in German. The study aims to analyze the short term effects of physical activities on L2 acquisition. It involves an experiment group contentbased PE lessons with an approach to L2 learning acquisition. The control group did not receive any treatment. Vocabulary, listening comprehension, and prepositions were the main indicator for pre- and post-test. The research found that the intervention group significantly performed better in vocabulary and listening comprehension compared to the control group.

A study by Padial-Ruz et.al [15] examining the relation of physical activity to teaching content in children. Similar to a previous study by mavilidi [12], this study integrated gesture and physical activity to 88 children L2 learning. It carried out a quasi-experimental research design under three conditions; a group of children learns L2 with gestures only, a group of children learn L2 with gesture and motor activity whereas the control group was taught using traditional method without any gesture or motor intervention. The study concluded that learning L2 with gesture and combination of gesture and motor activity promotes more effective learning of L2 vocabulary. However, the highest L2 acquisition achieved in the combined condition where children learn the language together with gesture and motor activities. However, this study did not examine the long-term effect of the intervention by conducting a delay test. Thus the effect on long-term memory remains unknown.

The author has tried to limit the study on the effect of the physical activities toward L2 learning. However, out of the expectation, most of the study employs the L2 only as the media to measure the cognitive level of participants during the physical activities intervention in terms of short-term and long-term memory. Vocabulary list and L2 memorization activity were utilized as an instrument to recall cognitive function. Thus, the papers discussed mostly on the effect of various exercises toward a very basic linguistic level, in this case, is vocabulary either common vocabulary or novel vocabulary $[5,9,10,12]$. Hence, it is unknown whether the condition of memorizing the L2 vocabulary able to lead the L2 learner to go higher in sentence creating level. One paper does discuss on the semantic level (comprehension of a sentence) but the study involves L2 learner who has a certain level of L2 proficiency, meaning that the L2 learner has been exposed to the language before and this may interfere with the semantic level of the subject [13]. Interestingly each study was taken into consideration of previous study being cited in the review, meaning that one is learning and examining other works in the same field.

The presented paper discussed in this review was incorporating various physical activities in each study namely high running exercise, team games, circuit training, gesture, and biking[5, 9-11]. The differences in learning outcomes for those different exercise types are reminded unknown. However, the trend showed that as long as the physical activities involve whole-body movement the correlation is more positive toward L2 learning. Hence, when it does involve part-body movement such as in gesturing activities on the sitting condition, the differences are significant. Students involved in whole-body activities perform significantly better for the free-recall test in all-time duration (during, immediately after) compared to those on part-body movement [12]. The question then comes to the research utilizing cycling activities as in Liu et. Al, and Schmidt-Kassow et. al [10, 13], isn't it similar to gesturing activities were only part of the body that is moving for spinning the bike. But the author did not discuss either explain the condition. Hence, they were categorizing cycling activity as continuous exercise during learning. 
Regarding the time of testing, most of the paper divided the testing phase into three main timelines, during, immediately after and several weeks after the treatment to find the correlation of physical activities toward the learning memory either short-term memory (during and immediately after the exercise) as well as long term memory. There is no consensus result for each phase but the overall trend showed that when it does positively affect the L2 vocabulary in the short-term memory, the L2 vocabulary test reminds the same for long term memory.

Studies concluded that physical activity stimulates brain activity which improves learning [10] which in turn makes it ideal to improve learning. Additional, simultaneous physical activities positively increase the ability to memorize new vocabulary[13] for beginner L2 learners. In contrast, to study by Liu [13] Study by Schmidt-Kassow and colleagues [10], Winter and colleagues [9] and the remaining study were given to a new learner of L2. This hinders the potential elaboration of research due to in the initial stage of learning language fluency is very limited.

In terms of gender, two out of eight studies involved men only $[9,16]$. Whereas study in children found the different results of physical activities intervention toward boys versus girls in various aspects including cognitive, measurement, working memory, and fitness performance $[4,17,18]$. Thus the two reminded papers $[9,16]$ should take more consideration on gender proportion if they want to maintain the generalization of the data.

\section{Conclusion}

The present systematic literature review found evidence to suggest that there is a positive association between physical activities and L2 learning. The positive effect is quite similar to children and adults either female or male. The finding is consistently positive for short-term memory and long-term memory in terms of vocabulary acquisition. Research that determining the integration of L2 in PA as a whole-body movement versus part-body movement tends to show a more positive result in L2 performance for the whole-body intervention. The physical activities employ for the studies are quite varied in terms of the time, activity, frequency, and amount. Thus, there is no standard of physical activities to enhance L2 performance. Hence, most of the research in the field focused on the very basic vocabulary acquisition, very limited has discussed the influence of PA in a deeper aspect of language acquisition such as listening, speaking, or writing. To the best of knowledge, the highest level being examined was in the semantic judgment. Based on the provided evidence, the author concluded that integrating physical activities in L2 learning bring a positive effect on cognition of L2 vocabulary, however, more research needs to be conducted to test the effect of physical activities toward other aspects of L2 learning such as listening and reading comprehension.

\section{References}

[1] Padulo J, Bragazzi NL, De Giorgio A, Grgantov Z, Prato S, Ardigò LP. The Effect of Physical Activity on Cognitive Performance in an Italian Elementary School: Insights From a Pilot Study Using Structural Equation Modeling. Frontiers in Physiology. 2019;10:202.

[2] Doherty A, Forés Miravalles A. Physical Activity and Cognition: Inseparable in the Classroom. Frontiers in Education. 2019;4:105. 
[3] Mandolesi L, Polverino A, Montuori S, Foti F, Ferraioli G, Sorrentino P, et al. Effects of Physical Exercise on Cognitive Functioning and Wellbeing: Biological and Psychological Benefits. Front Psychol. 2018;9:509-.

[4] Hillman ESDMRSLBRRDMMBPKIECH. The sexual dimorphic association of cardiorespiratory fitness to working memory in children. Developmental Science. 2015;19(1):90-108.

[5] Pesce C, Crova C, Cereatti L, Casella R, Bellucci M. Physical activity and mental performance in preadolescents: Effects of acute exercise on free-recall memory. Mental Health and Physical Activity. 2009;2(1):16-22.

[6] Moher D, Liberati A, Tetzlaff J, Altman DG, The PG. Preferred Reporting Items for Systematic Reviews and Meta-Analyses: The PRISMA Statement. PLOS Medicine. 2009;6(7):e1000097.

[7] Khan KS, Kunz R, Kleijnen J, Antes G. Five steps to conducting a systematic review. J R Soc Med. 2003;96(3):118-21.

[8] Wahono R. A Systematic Literature Review of Software Defect Prediction: Research Trends, Datasets, Methods and Frameworks. Journal of Software Engineering. 2015;1.

[9] Winter B, Breitenstein C, Mooren FC, Voelker K, Fobker M, Lechtermann A, et al. High impact running improves learning. Neurobiology of Learning and Memory. 2007;87(4):597-609.

[10] Schmidt-Kassow M, Kulka A, Gunter TC, Rothermich K, Kotz SA. Exercising during learning improves vocabulary acquisition: Behavioral and ERP evidence. Neuroscience Letters. 2010;482(1):40-4.

[11] Er S. TPR in FSL Early childhood education. Procedia - Social and Behavioral Sciences. 2013;93:1766-8

[12] Mavilidi M-FO, Anthony D.; Chandler, Paul A.; Cliff, Dylan P.; and Paas, Fred. Effects of integrated physical exercises and gestures on preschool. Education Psychology Review 2015

[13] Liu F, Sulpizio S, Kornpetpanee S, Job R. It takes biking to learn : Physical activity improves learning a second language. 2017:1-15.

[14] Krüger M. Second language acquisition effects of a primary physical education intervention: A pilot study with young refugees. PLoS One. 2018;13(9):e0203664-e.

[15] Padial-Ruz R, García-Molina R, Puga-González E. Effectiveness of a Motor Intervention Program on Motivation and Learning of English Vocabulary in Preschoolers: A Pilot Study. Behav Sci (Basel). 2019;9(8):84.

[16] Donnelly JE, Hillman CH, Castelli D, Etnier JL, Lee S, Tomporowski P, et al. Physical Activity, Fitness, Cognitive Function, and Academic Achievement in Children: A Systematic Review. Med Sci Sports Exerc. 2016;48(6):1197-222.

[17] Reed JA, Einstein G, Hahn E, Hooker SP, Gross VP, Kravitz J. Examining the impact of integrating physical activity on fluid intelligence and academic performance in an elementary school setting: a preliminary investigation. J Phys Act Health. 2010;7(3):343-51.

[18] Reed JA MA, Long S, Hughey M. Examining the impact of 45 minutes of daily physical education on cognitive ability, fitness performance, and body composition of African American youth. Journal of physical activity \& health. 2013;10(2):185-97. 